\title{
Mass spectra of the low-lying nonet scalar mesons in the lattice QCD
}

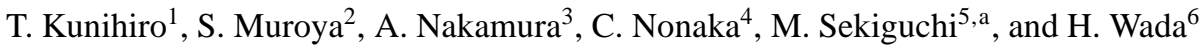 \\ 1 Department of Physics, Kyoto University Kyoto, 606-8502, Japan \\ 2 Department of Comprehensive Management, Matsumoto University, Matsumoto 390-1295, Japan \\ 3 RIISE, Hiroshima University, Higashi-Hiroshima 739-8521, Japan \\ 4 Department of Physics, Nagoya University, Nagoya 464-8602, Japan \\ 5 Department of Mathematics and Science, Kokushikan University,Tokyo 154-8515, Japan \\ 6 Faculty of Political Science and Economics, Kokushikan University, Tokyo 154-8515, Japan
}

\begin{abstract}
We review the works done by our collaboration (Scalar collaboration) for the scalar mesons in the lattice QCD. We present the preliminary results and the plan for simulation of the sigma meson and the kappa meson.
\end{abstract}

\section{Introduction}

The nature of the low-lying nonet scalar mesons continues to be an interesting problem in hadron physics. The existence of the $I=0$ and $J^{P C}=0^{++}$meson, i.e., the $\sigma(400-600)$ has been re-confirmed [3] after some twenty years not only in $\pi \pi$ scattering $[1,2]$ but also in various decay processes from heavy-quark systems e. g. , $\mathrm{D} \rightarrow \pi \pi \pi$ and $\Upsilon(3 S) \rightarrow \Upsilon \pi \pi$ [4-7]. Moreover, a resonance of scalar meson with $I=1 / 2$ is also reported to exist in the $\mathrm{K}-\pi$ system with a mass $m_{\kappa}$ of about $800 \mathrm{MeV}[8,9,7]$. This meson is called the $\kappa$ and may constitute the nonet scalar state together with the $\sigma$ meson. See Fig.1.

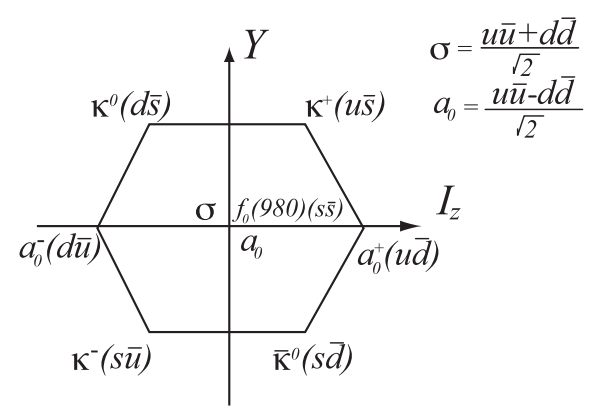

Fig. 1. Scalar meson nonet. The $\sigma$ and $f_{0}(980)$ mesons may be ideal mixing states of singlet state $\frac{1}{\sqrt{3}}(u \bar{u}+d \bar{d}+s \bar{s})$ and octet state $\frac{1}{\sqrt{6}}(u \bar{u}+d \bar{d}-2 s \bar{s})$. There is , however, experimental evidence that the $\sigma$ meson consist of only $u \bar{u}$ and $d \bar{d}$ components. Hence, we take the $\sigma$ wave function given in the figure.

The problem is the nature of these low-lying scalar mesons [10]: they can not be usual $q \bar{q}$ mesons as described

a e-mail: motoo@kokushikan.ac.jp in the non-relativistic costituent quark model since in such a quark model, $J^{P C}=0^{++}$meson is realized in the ${ }^{3} P_{0}$ state, which implies that the mass of the $\sigma$ meson must be as high as $1.2 \sim 1.6 \mathrm{GeV}$. So the low-lying scalar mesons below 1 $\mathrm{GeV}$ has been a source of various ideas of exotic structures, as mentioned above: they may be four quark states like $q q \bar{q} \bar{q}[11,12]$, or $\pi \pi$ or $\mathrm{K} \pi$ molecules and so on as the recent high-lying exotic hadrons can be. These mesons may be collective $q \bar{q}$ states described as a superposition of many atomic $q \bar{q}$ states $[13,14]$. A mixing with glueball states is also possible [15-17].

Now there have been a number of simulations of lattice QCD for clarifying the structure of the low-lying scalar mesons such as the $\sigma$ and $\kappa$. It is noteworthy that elucidating the mechanism for these scalar mesons to have such a low mass in terms of their structures would also give a good clue to the possible structure of the exotic hadrons with heavy quark.

In this paper, we report our approaches to the $\sigma$ meson and the $\kappa$ meson.

\section{The sigma meson in the lattice QCD simulation}

There have been several attempts at lattice study of the $\sigma$ mesons. To our knowledge, the first such calculation was carried out by DeTar and Kogut [18], where the so-called disconnected diagram, or the OZI forbidden type diagram is discarded. The channel was called valence sigma, $\sigma_{v}$. They measured the screening masses and observed that $\sigma_{v}$ is much heavier than the $\pi$ meson at zero temperature, while the $\sigma_{v}$ and the $\pi$ degenerate as the temperature increases over $T_{c}$. Kim and Ohta calculated lattice the mass of $\sigma_{v}$ with staggered fermions for the lattice spacing $a=$ $0.054 \mathrm{fm}$ and lattice size $48 a=2.6 \mathrm{fm}$ [19]. They obtained $m_{\sigma_{v}} / m_{\pi}=1.4 \sim 1.6$ by varying $m_{\pi} / m_{\pi}=0.65 \sim 0.3$. 
Lee and Weingarten [15] have stressed the importance of the importance of the mixing the scalar meson and the glueballs and concluded that $f_{0}(1710)$ is the lightest scalar glueball dominant particle, while $f_{0}(1390)$ is composed of mainly the $u$ and $d$ quarkonium. Alfold and Jaffe analyzed the possibility that the $\sigma$ is an exotic state i.e., tetraquark $(q q \bar{q} \bar{q})$ by a quenched lattice QCD discarded the disconnected diagram [12]. Riken-Brookhaven-Columbia (RBC) collaboration [33] employed the domain wall fermions for the lattice study of the $\sigma$, which respected the chiral symmetry, but include a quench a quench approximation with the help of the chiral perturbation. All these calculations are in the quench approximation, i.e., the fermion determinant is dropped, which corresponds to ignoring quark pair creation and annihilation diagrams. UKQCD observed that the $\sigma$ meson masses in the quench approximation and in the full QCD simulation are very different [16]. They also considered the mixing with the glueballs. They obtained a very small $\sigma$ meson mass, even smaller than the $\pi$ mass, in the full QCD case.

Our collaboration [21-24] performed a exploratory work on the $\sigma$ in the full lattice QCD simulation. We remark that the full QCD simulation is necessary to properly describe the $\sigma$ with the possible contents, i.e., the glueball, terra quarks and so on.

The quantum numbers of the $\sigma$ meson are $I=0$ and $J^{P C}=0^{++}$; We adopt the $\sigma$ meson operator as

$$
\hat{\sigma}(x) \equiv \sum_{c=1}^{3} \sum_{\alpha=1}^{4} \frac{\bar{u}_{\alpha}^{c}(x) u_{\alpha}^{c}(x)+\bar{d}_{\alpha}^{c}(x) d_{\alpha}^{c}(x)}{\sqrt{2}}
$$

where $u$ and $d$ indicate the corresponding quark spinors. Indices $c$ and $\alpha$ stand for color and Dirac spinor indices, respectively. The $\sigma$ meson propagator is written as

$$
\begin{aligned}
G(y, x)= & <\mathrm{T} \hat{\sigma}(y) \hat{\sigma}(x)^{\dagger}> \\
= & \frac{1}{Z} \int D U D \bar{u} D u D \bar{d} D d \\
& \sum_{a, b=1}^{3} \sum_{\alpha, \beta=1}^{4} \frac{\bar{u}_{\beta}^{b}(y) u_{\beta}^{b}(y)+\bar{d}_{\beta}^{b}(y) d_{\beta}^{b}(y)}{\sqrt{2}} \\
\times \quad & \left(\frac{\bar{u}_{\alpha}^{a}(x) u_{\alpha}^{a}(x)+\bar{d}_{\alpha}^{a}(x) d_{\alpha}^{a}(x)}{\sqrt{2}}\right)^{\dagger} e^{-S_{G}-\bar{u} W u-\bar{d} W d} .
\end{aligned}
$$

In Eq.(2), $W^{-1}$ 's are $u$ and $d$ quark propagators, $U$ 's are link variables of gluon, and $S_{G}$ is the gauge action. By integrating over $u, \bar{u}, d$ and $\bar{d}$ fields, the $\sigma$ meson propagator is given by

$$
\begin{aligned}
G(y, x)= & -<\operatorname{Tr} W^{-1}(x, y) W^{-1}(y, x)> \\
& +2<(\sigma(y)-<\sigma(y)>) \\
& \cdot(\sigma(x)-<\sigma(x)>)>
\end{aligned}
$$

where

$$
\sigma(x) \equiv \operatorname{Tr} W^{-1}(x, x) .
$$

"Tr" represents summation over color and Dirac spinor indices. Here we assumed that the $u$ and $d$ quark propagators are equivalent because $u$ and $d$ quark masses are almost the same. From Eq.(3), we can see that the $\sigma$ propagator consists of two terms. The first term corresponds to the connected diagram, i.e., $\bar{q} q$ type meson. The second term comes from the disconnected diagram, i.e., the correlation of fluctuation of the operator $\bar{q}(x) q(x)$. The quantum number of the $\sigma$ meson $\left(I=0\right.$ and $\left.J^{P}=0^{+}\right)$is the same as that of the vacuum, and the vacuum expectation value of the $\sigma$ operator, $\langle\sigma(x)>$ does not vanish. Therefore, the contribution of $\langle\sigma(x)>$ should be subtracted from the $\sigma$ operator.

We employ Wilson fermions and the plaquette gauge action. The dynamical quarks effects are expected to be important in estimation of the disconnected diagrams of the $\sigma$ propagator. Therefore, the $\sigma$ propagator should be calculated in the full QCD simulation. For this purpose, we generated the gauge configurations by using Hybrid Monte Carlo (HMC) algorithm.

CP-PACS performed a very large scale simulation of the light meson spectroscopy in full QCD calculation [25]. We use here the same values of the simulation parameters, i.e. $\beta=4.8$ and the using hoping parameter $h=0.1846$, $0.1874,0.1891$ except lattice size; Our lattice size, $8^{3} \times 16$, is smaller than theirs. Note that we employ the point source and sink, and smaller lattice size results in larger mass due to higher state mixture. In other words, our mass values on the small size lattice should be considered as an upper limit. We show in Table 1 the value of $m_{\pi} / m_{\rho}$ together with that of CP-PACS. They are consistent within error bar. We generated the gauge configurations in full QCD by using HMC algorithm. First 1500 trajectories were updated in quenched QCD, then we switch on the dynamical fermion. Next 2000 trajectories of HMC are discarded as the thermalization a nd the $\sigma, \pi$ and $\rho$ propagators are calculated in every ten trajectories. It is very hard to evaluate the disconnected part of the propagator, since we must calculate $\operatorname{Tr} W^{-1}(x, x)$ for all lattice sites $x$. We used the $Z_{2}$ noise method to calculate the disconnected diagrams and the subtraction terms of the vacuum $\langle\sigma\rangle$. Each of these terms is the order of ten, and $(\sigma-\langle\sigma\rangle)(\sigma-\langle\sigma\rangle)$ becomes less than $10^{-4}$. Therefore high accuracy is required for the calculation. One thousand random $Z_{2}$ numbers are generated. Our numerical results show that the values of the second in Eq.(3) are in the same order. Therefore, in order to obtain the signal correctly as the difference between these terms, the high precision numerical simulations and careful analyses are essential. The relation between the number of $Z_{2}$ noise and the achieved accuracy was investigated in Ref.[21]. Gauge configurations were created by HMC in SX5 vector super computer, and most disconnected propagator calculations by $Z_{2}$ noise method were mainly performed SR8000 parallel machine.

We show in Table 1 the value of $m_{\sigma} / m_{\rho}$ for each hopping parameter together with the corresponding $m_{\text {con. }} / m_{\rho}$, where $m_{\text {con. }}$ denotes the scalar meosn mass for which the disconnected diagram is not included. It should be noticed here that the mass $m_{c o n}$. can be identified with the $\mathrm{a}_{0}$ meson which is the isovector isoscalar meson for which the disconnected diagrams does not play any role.

The individual contributions of the connected and disconnected parts of the $\sigma$ propagator are shown in Fig.2, 
Table 1. Summary of the results.

\begin{tabular}{llll}
\hline$h$ & 0.1846 & 0.1874 & 0.1891 \\
\hline statistics & 1110 & 860 & 730 \\
$m_{\pi} / m_{\rho}($ CP-PACS $)$ & $0.8291(12)$ & $0.7715(17)$ & $0.7026(32)$ \\
$m_{\pi} / m_{\rho}($ Scalar $)$ & $0.825(2)$ & $0.757(2)$ & $0.693(3)$ \\
$m_{\sigma} / m_{\rho}($ Scalar $)$ & $1.6(1)$ & $1.34(8)$ & $1.11(6)$ \\
$m_{\text {connect }} / m_{\rho}($ Scalar $)$ & $2.40(2)$ & $2.44(3)$ & $2.48(4)$ \\
\hline
\end{tabular}

which tells us that the connected part only shows a rapid damping with small error bars, while the disconnected part overwhelming the connected part and dominates the $\sigma$ propagator. Thus, we see that the $\sigma$ as a light meson results from the disconnected part of the $\sigma$ propagator with the background vacuum condensate subtracted.

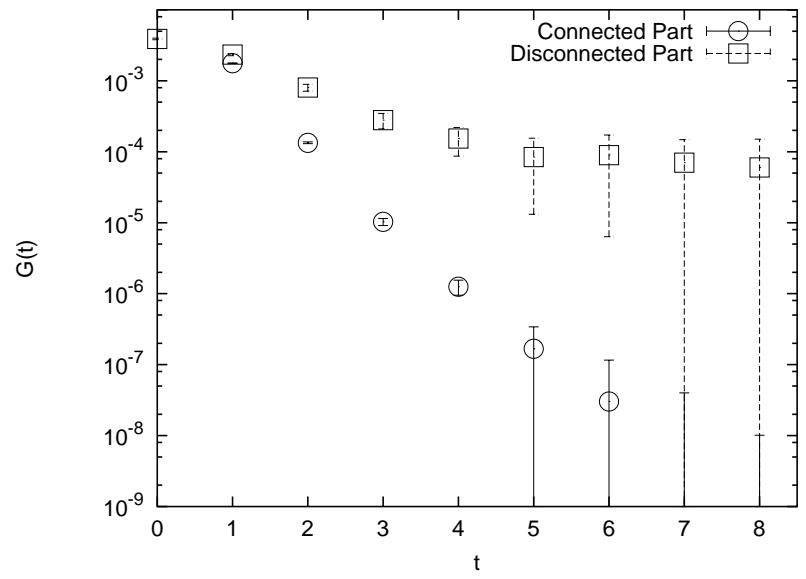

Fig. 2. Propagators of the connected and disconnected diagrams of the $\sigma$ for $\kappa=0.1874$.

The calculated $m_{\pi}^{2}, m_{\rho}, m_{\sigma}$ and $2 m_{\pi}$ are presented in Fig. 3 of ref.[24], which shows that as the chiral limit is approached, the $\sigma$ meson mass obtained from the $\sigma$ propagator decreases and eventually becomes smaller than the $\rho$ meson mass in the chiral limit.

It might be more informative to display the masses as functions of $m_{\pi}^{2}$, which could be extracted by some effective models[20]. The $m_{\pi}^{2}$ dependence of $m_{\sigma}$ and $m_{\rho}$ is shown in Fig.3, which is equivalent to Fig.3 of ref.[24].

Important points obtained in ref. [24] are that the $\sigma$ propagator exhibits a pole behavior and its mass is found to satisfy $m_{\pi}<m_{\sigma} \leq m_{\rho}$; for the sigma mass to become small, the disconnected diagram plays an essential role. The flavored scalar meson is not light as observed experimentally; $m_{a_{0}} \sim 1.9 \mathrm{GeV}$, which are much higher than the experimental masses, $0.6 \sim 0.8 \mathrm{GeV}$.

The UKQCD collaboration [26], used the full lattice QCD, with a variational fit with glue and $\bar{q} q$ interpolating operators, to study the $\sigma$ meson. The mass of the $\sigma$ is below $1 \mathrm{GeV}$.

Some lattice groups [27-29] maintained to get result for the mass of sigma using the tetraquark type interpolating operator from the quenched lattice QCD. Recently,

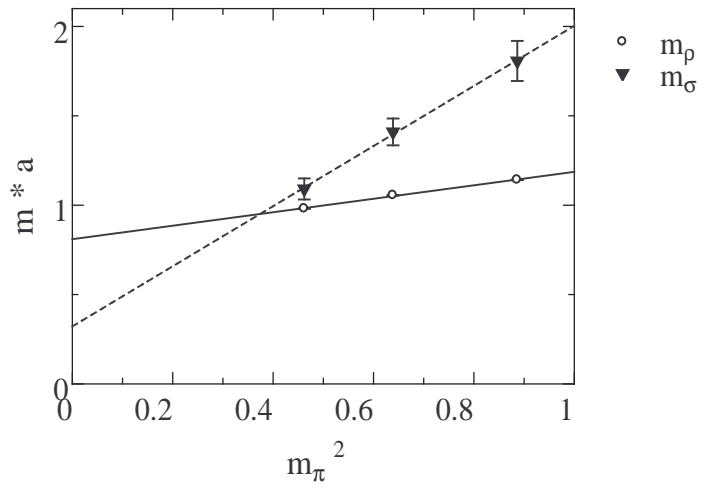

Fig. 3. The $m_{\pi}^{2}$ dependence of $m_{\rho}$ and $m_{\sigma}$ in the lattice unit. Explicitly, the respective dependence is well represented by a linear function of $m_{\pi}^{2}: m_{\rho} a=0.3767 \times\left(m_{\pi} a\right)^{2}+0.8099$ and $m_{\sigma} a=1.6818 \times\left(m_{\pi} a\right)^{2}+0.3219$, respectively, with $a$ being the lattice spacing.

Prelovsek et al. [30] found the light state in the sigma channel using tetraquark type interpolating operator discarded the disconnected diagrams from the quenched and the full lattice QCD. The disconnected diagram of these results needs to be quantified.

\section{The kappa meson in the lattice QCD simulation}

At the first we performed a lattice calculation of the $\kappa$ meson in the full QCD simulation using the dynamical fermion for the $u(d)$ quark but the valence approximation for the $s$ quark on the $8^{3} \times 16$ lattice with the plaquette action and Wilson fermions[31,24]. We have presented that the $\kappa$ meson has a mass as large as about $1.8 \mathrm{GeV}$ and can not be identified with the $\kappa$ meson seen in experiments. The lattice volume in the previous investigations was admittedly small to have definite conclusions at all, and the lattice cutoff was not appropriately chosen to accommodate the large masses $m_{\kappa} a>1$, where $a$ is the lattice spacing. We have performed the quenched simulations on the $\kappa$ meson[32] so as to clarify the structure of the scalar meson rather than to reproduce the experimental value of the mass; a quenchedlevel simulation should give a rather clear perspective on whether the system can fit with the simple quark model picture or not. For comparison, we also perform a simulation on the axial vector meson $\left(K_{1}\right)$ with $I=1 / 2 J^{P}=1^{+}$, which is found to well describe the physical system even in the quenched level: the $K_{1}$ comes from the mixing between $I=1 / 2 J^{P C}=1^{++}$and $1^{+-}$. In fact, the axial vector meson $K_{1 A}$ and $K_{1 B}$ are mixtures of the $K_{1}(1270)$ and $K_{1}(1400)$. Hence, our simulation for the axial vector meson is an ideal case. Moreover, we also present the result on the valence $\sigma$ meson for getting into the intuition on the sturucture of the scalar mesons. We have used the Wilson fermion with the plaquette gauge action, on a relatively large lattice, i.e., $20^{3} \times 24$. The values of the hopping parameter for the $u / d$ quark are $h_{u / d}=0.1589,0.1583$ and 0.1574 , while $h_{s}=0.1566$ and 0.1557 for the $s$ quark. Using these 
Table 2. Summary of results for $\bar{q} q$ type mesons.

\begin{tabular}{llll}
\hline \hline Scalar[32] & & & \\
\hline \hline$h_{u / d}$ & $m_{\pi}$ & $m_{\rho}$ & $m_{\pi} / m_{\rho}$ \\
\hline 0.1589 & $0.2064(62)$ & $0.442(13)$ & $0.467(21)$ \\
0.1583 & $0.2691(36)$ & $0.461(06)$ & $0.584(10)$ \\
0.1574 & $0.3401(29)$ & $0.496(05)$ & $0.686(05)$ \\
0.1566 & $0.3935(28)$ & $0.527(04)$ & $0.746(03)$ \\
0.1557 & $0.4478(28)$ & $0.563(03)$ & $0.796(03)$ \\
\hline CP-PACS[35] & & & \\
\hline \hline$h_{u / d}$ & $m_{\pi}$ & $m_{\rho}$ & $m_{\pi} / m_{\rho}$ \\
\hline 0.1589 & $0.20827(33)$ & $0.42391(132)$ & $0.491(2)$ \\
0.1583 & $0.26411(28)$ & $0.44514(96)$ & $0.593(1)$ \\
0.1574 & $0.33114(26)$ & $0.47862(71)$ & $0.692(1)$ \\
0.1566 & $0.38255(25)$ & $0.50900(60)$ & $0.752(1)$ \\
\hline & & & \\
Scalar[32] & & & \\
\hline \hline$h_{u / d}$ & $m_{\sigma_{v}}$ & $m_{a_{1}}$ & \\
\hline 0.1589 & $1.12(74)$ & $0.862(76)$ & \\
0.1583 & $0.84(23)$ & $0.895(48)$ & \\
0.1574 & $0.886(98)$ & $0.913(32)$ & \\
0.1566 & $0.857(52)$ & $0.932(24)$ & \\
0.1557 & $0.893(35)$ & $0.959(20)$ & \\
\hline
\end{tabular}

hopping parameters except for $h_{s}=0.1557$, CP-PACS collaboration performed a quenched QCD calculation of the light meson spectrum with a larger lattice $\left(32^{3} \times 56\right)$ [35], which we refer to for comparison. The gauge configurations are generated by the heat bath algorithm at $\beta=5.9$. After 20000 thermalization iterations, we start to calculate the meson propagators. On every 2000 configurations, 80 configurations are used for the ensemble average.

We emply the point-like source and sink for the $\kappa^{+}$meson

$$
\hat{\kappa}(x) \equiv \sum_{c=1}^{3} \sum_{\alpha=1}^{4} \bar{s}_{\alpha}^{c}(x) u_{\alpha}^{c}(x),
$$

where $u(x)$ and $s(x)$ are the Dirac operators for the $u / d$ and $s$ quarks, and the indices $c$ and $\alpha$ denote the color and Dirac-spinor indices, respectively. The point source and sink in Eq.(5) lead a positive spectral function $\rho\left(\mathrm{m}^{2}\right)$ in the correlation function $\langle\hat{\kappa}(t) \hat{\kappa}(0)\rangle=\int d m \rho\left(m^{2}\right) \exp (-m t)$. The result obtained here is thus an upper bound of $\kappa$ mass, because our result should include excited states.

First, we check finite lattice volume effects by comparing our results for the $\pi$ and $\rho$ masses as well as the mass ratio $m_{\pi} / m_{\rho}$ with those of the CP-PACS group. The results are summarized in Table 2. Our result for the $\rho$ meson mass is only slightly $(<5 \%)$ larger than the CP-PACS's result. The resulting larger value is reasonable because the smaller lattice size gives rise to a mixture of higher mass states. We rather emphasize that the deviation between our results and the larger lattice result (CP-PACS) is so small in spite of the large difference in the lattice size.

In Fig. 4, we display $m_{\pi}^{2}, m_{\rho}, m_{\sigma_{v}}$ and $m_{a_{1}}$ in the lattice unit as a function of the inverse hopping parameter $1 / h_{u / d}$ for the $u / d$ quark. We see that the chiral limit $\left(m_{\pi}^{2}=0\right)$ is obtained at $h_{u / d}=0.1598(1) \equiv h_{\text {crit }}\left(1 / h_{\text {crit }}=6.2581\right)$. We find the lattice spacing $a=0.1038(33)[\mathrm{fm}]$ in the chiral limit from the value $m_{\rho} a=0.406(13)$ at this point with the physical $\rho$ meson mass being used for $m_{\rho}$. We remark that these values are consistent with the CP-PACS's result, $h_{\text {crit }}$ $=0.1598315(68)$ and $a=0.1020(8)[\mathrm{fm}]$, within the error bars. Again, our smaller-lattice simulation well reproduces the results in the larger lattice.

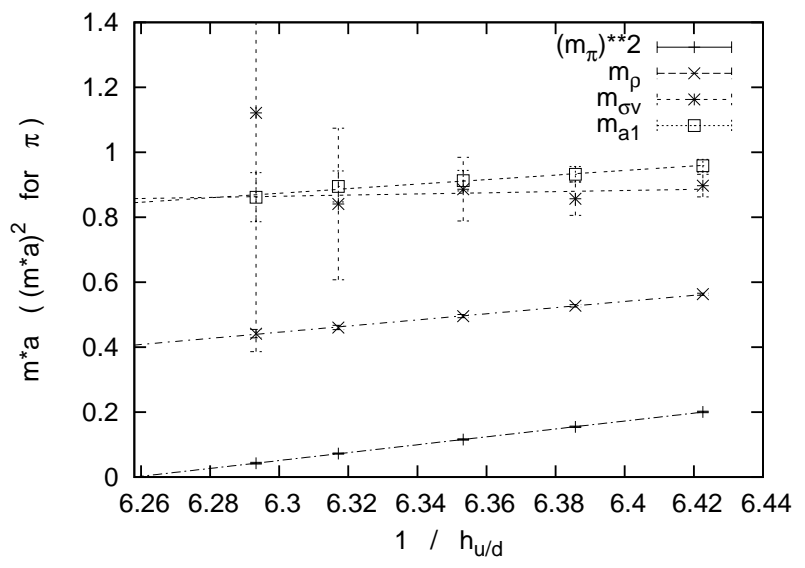

Fig. 4. $m_{\pi}^{2}, m_{\rho}, m_{\sigma_{v}}$ and $m_{a_{1}}$ in the lattice unit as a function of the inverse $h_{u / d}$. The chiral limit is obtained at $h_{\text {crit }}=0.1598(1)$.

In Table 2, masses of valence $\sigma\left(a_{0}\right)$ for each hopping parameter are shown. $m_{\sigma_{v}} / m_{\rho}$ varies from $2.5\left(h_{u / d}=\right.$ $0.1589)$ to $1.6\left(h_{u / d}=0.1557\right)$ which is consistent with our previous results [24]. In other words, without disconnected part of the propagator the mass of " $\sigma$ " becomes heavy. We also present $a_{1}$ mass in Table 2 .

Now the propagators of the $K, K^{*}, \kappa$ and $K_{1}$ mesons are calculated with the same configurations using the $s$ quark hopping parameter, $h_{s}=0.1566$ and 0.1557 . For $h_{s}$ $=0.1557$, the effective mass plots of $K^{*}, \kappa$ and $K_{1}$ mesons are shown in Figs. 5-7. The masses of the $K, K^{*}, \kappa$ and $K_{1}$ which are extracted from the effective mass plots are summarized in Tables 3 and 4. We find that the effective masses of the $K$ and $K^{*}$ mesons have only small errors and are taken to be reliable, while those of the $\kappa$ and $K_{1}$ suffer from large errors, especially at larger time. To avoid possible large errors coming from the data at large $t$, we fit the effective masses of the $\kappa$ and $K_{1}$ only in the time range $5 \leq t \leq$ 7,8 where the effective masses are almost constant with small errors. Since the effective mass of $K^{*}$ is reliable, we show the $\kappa$ and $K_{1}$ masses in terms of the ratio to $m_{K^{*}}$ : table 5 gives the mass ratios $m_{K} / m_{K^{*}}, m_{K} / m_{K^{*}}$ and $m_{K_{1}} / m_{K^{*}}$ at the chiral limit together with $m_{\phi} / m_{K^{*}}$ for $h_{s}=0.1566$ and 0.1577. For example, $m_{\kappa} / m_{K^{*}}=0.89(29) / 0.4649(69)=$ $1.92(61)$ at $h_{s}=0.1566$ in Table 5. These calculated mass ratios are shown in Fig. 8. One can see that the all mass ratios are almost independent of the values of $h_{s}$. However error bar for the ratios $m_{\kappa} / m_{K^{*}}$ and $m_{K_{1}} / m_{K^{*}}$ is large, those behavior as a function of $h_{s}$ is reasonable.

Now we have searched the physical value of the $s$ quark hopping parameter $h_{s}$ in the following two ways, 
$19^{\text {th }}$ International IUPAP Conference on Few-Body Problems in Physics

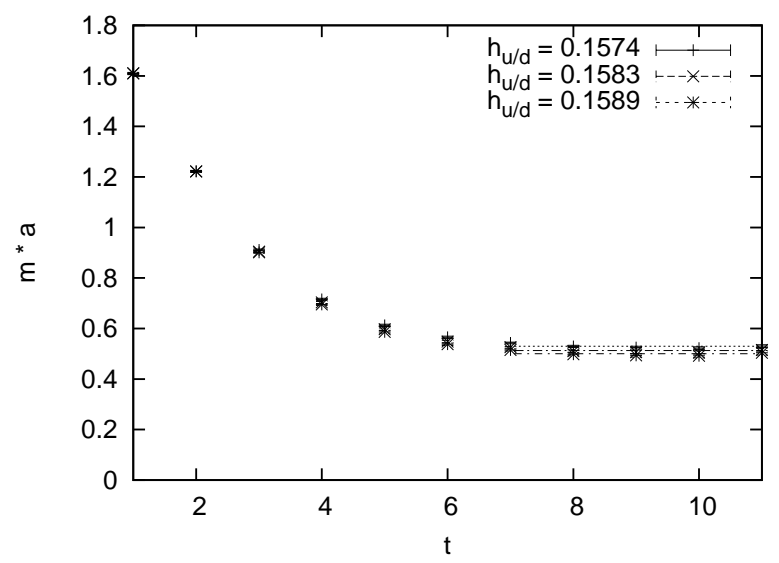

Fig. 5. Effective mass plots of $K^{*}$ for $s$ quark hopping parameter $h_{s}=0.1557$.

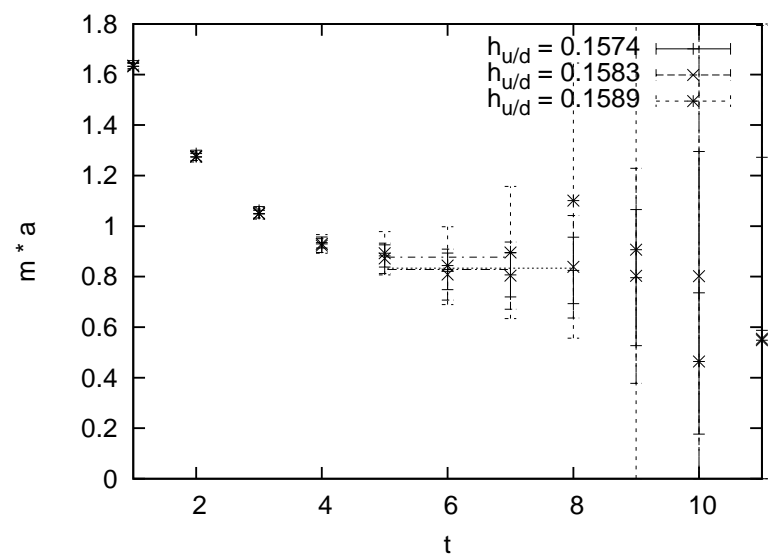

Fig. 6. Effective mass plots of $\kappa$ for $s$ quark hopping parameter $h_{s}$ $=0.1557$.

Table 3. The summary results for $K, K^{*}, \kappa$ and $K_{1}$ mesons at $h_{s}=0.1566$.

\begin{tabular}{c|c|c|c|c}
\hline \hline$h_{u / d}$ & $h_{\text {crit }}^{1)}$ & 0.1589 & 0.1583 & 0.1574 \\
\hline$m_{K}$ & $0.2829(23)$ & $0.3138(33)$ & $0.3368(30)$ & $0.3677(29)$ \\
$m_{K^{*}}$ & $0.4649(69)$ & $0.4821(57)$ & $0.4941(49)$ & $0.5117(42)$ \\
$m_{K}$ & $0.89(29)$ & $0.88(23)$ & $0.81(12)$ & $0.814(81)$ \\
$m_{K_{1}}$ & $0.919(43)$ & $0.922(36)$ & $0.915(32)$ & $0.922(27)$ \\
\hline \multicolumn{5}{|c}{ CP-PACS [35] } \\
\hline \hline$m_{K}$ & - & $0.30769(28)$ & $0.32833(26)$ & - \\
$m_{K^{*}}$ & - & $0.46724(84)$ & $0.47749(74)$ & - \\
\hline
\end{tabular}

1) $h_{\text {crit }}=0.1598(1)$.

both of which are found to give similar results: 1) By tracing a regression line for $m_{\phi} / m_{K^{*}}$ (Fig. 8), we have $h_{s}=$ $0.1563(3)\left(\right.$ or $\left.1 / h_{s}=6.396(13)\right)$ for $m_{\phi} / m_{K^{*}}=1019[\mathrm{MeV}]$ $/ 892.0[\mathrm{MeV}]=1.143$ (input), taken from the PDG [3]. This hopping parameter gives the mass ratios $\left(m_{\kappa} / m_{K^{*}}\right.$, $\left.\left.m_{K_{1}} / m_{K^{*}}\right)=(1.89(55), 1.962(78)) .2\right)$ We have also determined the hopping parameter so as to reproduce the mass ratio $m_{K} / m_{K^{*}}=495.6[\mathrm{MeV}] / 892[\mathrm{MeV}]=0.5556$, with

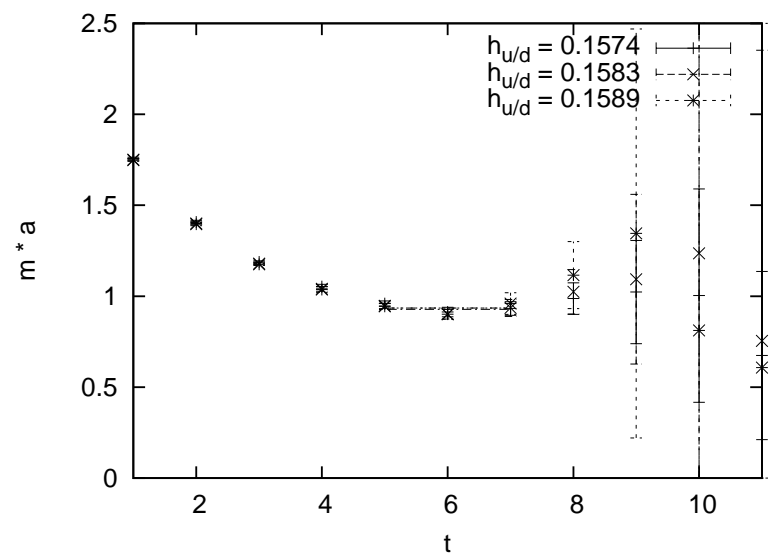

Fig. 7. Effective mass plots of $K_{1}$ for $s$ quark hopping parameter $h_{s}=0.1557$.

Table 4. The summary results for $K, K^{*}, \kappa$ and $K_{1}$ mesons at $h_{s}=0.1557$.

\begin{tabular}{c|c|c|c|c}
\hline \hline$h_{u / d}$ & $h_{\text {crit }}^{1)}$ & 0.1589 & 0.1583 & 0.1574 \\
\hline$m_{K}$ & $0.3188(25)$ & $0.3474(31)$ & $0.3684(29)$ & $0.3971(28)$ \\
$m_{K^{*}}$ & $0.4835(61)$ & $0.5006(52)$ & $0.5126(44)$ & $0.5299(37)$ \\
$m_{K}$ & $0.89(21)$ & $0.88(16)$ & $0.828(96)$ & $0.833(72)$ \\
$m_{K_{1}}$ & $0.931(36)$ & $0.935(30)$ & $0.928(26)$ & $0.935(23)$ \\
\hline
\end{tabular}

1) $h_{\text {crit }}=0.1598(1)$.

Table 5. The summary results for the mass ratios, $m_{K} / m_{K^{*}}$, $m_{\kappa} / m_{K^{*}}$ and $m_{K_{1}} / m_{K^{*}}$ together with $m_{\phi} / m_{K^{*}}$ at chiral limit for $u / d$ quarks.

\begin{tabular}{c|c|c||c|c}
\hline \hline$h_{s}$ & 0.1566 & 0.1557 & $0.1568(3)$ & $0.1576(2)$ \\
$1 / h_{s}$ & 6.3857 & 6.4226 & $6.396(13)$ & $6.3452(80)$ \\
\hline$m_{\phi} / m_{K^{*}}$ & $1.135(10)$ & $1.164(10)$ & $1.143^{1)}$ & - \\
$m_{K} / m_{K^{*}}$ & $0.6086(79)$ & $0.6593(63)$ & $0.623(11)$ & $0.5556^{1)}$ \\
$m_{\kappa} / m_{K^{*}}$ & $1.92(61)$ & $1.84(43)$ & $1.89(55)$ & $2.00(80)$ \\
$m_{K_{1}} / m_{K^{*}}$ & $1.977(86)$ & $1.926(69)$ & $1.962(78)$ & $2.03(10)$ \\
\hline
\end{tabular}

1) inputs for calculation of physical value of $h_{s}$. See the text.

$m_{K}=495.6[\mathrm{MeV}]$ being the average value of the Kaon masses given in the PDG [3]. The resulting value is found to be $h_{s}=0.1576(2)\left(\right.$ or $\left.1 / h_{s}=6.3452(80)\right)$, which in turn gives the mass ratio $m_{\kappa} / m_{K^{*}}=2.00(80)$ and $m_{K_{1}} / m_{K^{*}}=$ 2.03(10). The values of mass ratios which are obtained from two ways 1) and 2) are also presented in Table 5. One can see that both the ways give almost identical results for the masses of $\kappa$ and $K_{1}$ which are about twice of $K^{*}$ mass.

Prelovsek et al. [33] have presented a rough estimate of the mass of the $\kappa$ as $1.6 \mathrm{GeV}$ obtained using the average quark mass of the $u$ and $s$ quarks from the dynamical simulations with the degenerate $N_{f}=2$ quarks on a $16^{3} \times 32$ lattice. The UKQCD Collaboration have quite recently studied the $\kappa$ using the dynamical $N_{f}=2$ sea quarks and a valence strange quark on a $16^{3} \times 32$ lattice [34]; they estimated the $\kappa$ mass as about $1.1 \mathrm{GeV}$, much smaller than those in $[24,31,33]$ but still away from the experimental 


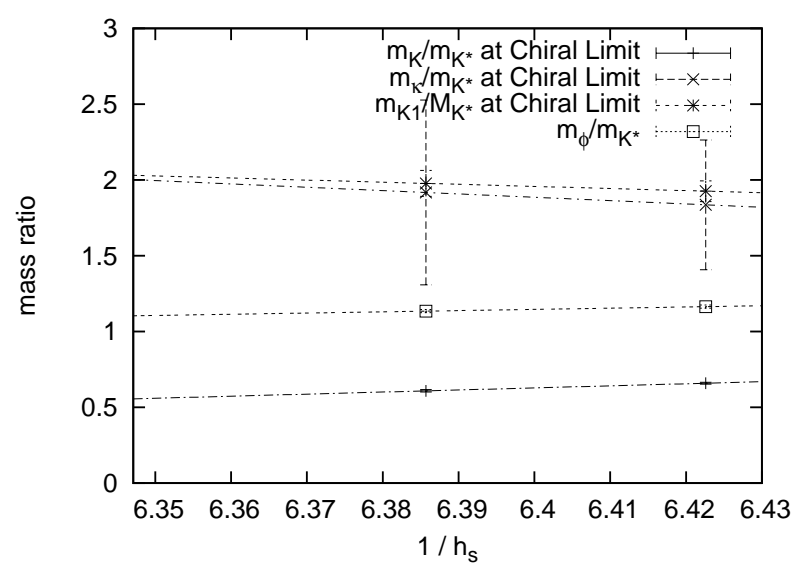

Fig. 8. The ratios $m_{K} / m_{K^{*}}, m_{\kappa} / m_{K^{*}}$ and $m_{K_{1}} / m_{K^{*}}$ at chiral limit, and $m_{\phi} / m_{K^{*}}$ for $s$ quark hopping parameters $h_{s}=0.1566$ and 0.1557 .

Table 6. The results for $\kappa$ meson.

\begin{tabular}{lc}
\hline \hline & $m_{\kappa} \mathrm{GeV}$ \\
\hline Scalar[31,24] & $\sim 1.7$ \\
Scalar[32] & $\sim 1.6$ \\
Prelovsek et al. [33] & $1.6(0.2)$ \\
UKQCD [34] & $1.1 \sim 1.2$ \\
Mathur et al. [27] & $1.41(0.12)$ \\
\hline
\end{tabular}

value $\sim 800 \mathrm{MeV}$. Mathur et al. [27] presented the results of the $\kappa$ channel using the type of tetraquark operator discarded the disconnected diagrams from the quench approximation. This result is consistent with experimental mass of the $K_{0}^{*}$ (1430). Recently, Prelovsek et al. [30] found the light state in the $\kappa$ channel using tetraquark type interpolating operator discarded the disconnected diagrams from the quenched and the full lattice QCD.

\section{Current status of our simulation for the scalar mesons}

We present a new simulation on large lattice $\left(16^{3} \times 32\right)$ than our previous simulation in full QCD. We will adopt several interpolating operators ( $q \bar{q}, q \bar{q} q \bar{q}, q q \bar{q} \bar{q}$, gluon etc.) for the scalar mesons by applying covariant derivatives on Jacobi smeared sources and sinks [36]. We employ the all to all propagator to improve the statistical accuracy. We use gauge configuration from CP-PACS collaboration [37]. These configurations were generated with renormalizationgroup improved action and the Wilson-clover quark action. Our simulations are the preliminary stages. As test simulations, Fig. 9 and Fig. 10 shows the propagator of the $\kappa$ meson using the smeared sources and sinks in the time direction. We use here the simulation parameters, i.e. $\beta=1.95$ and the using $u / d$ quark hoping parameter $h_{u / d}=0.1390$, $s$ quark hopping parameter $h_{s}=0.1386, C_{s w}=0.15300$. 20 configurations are used. It is necessary to improve the statistical precision of the estimation of the $\kappa$ propagator.
We also plan to improved the $\sigma$ propagator using the all to all propagator with the dilution techniques [38].

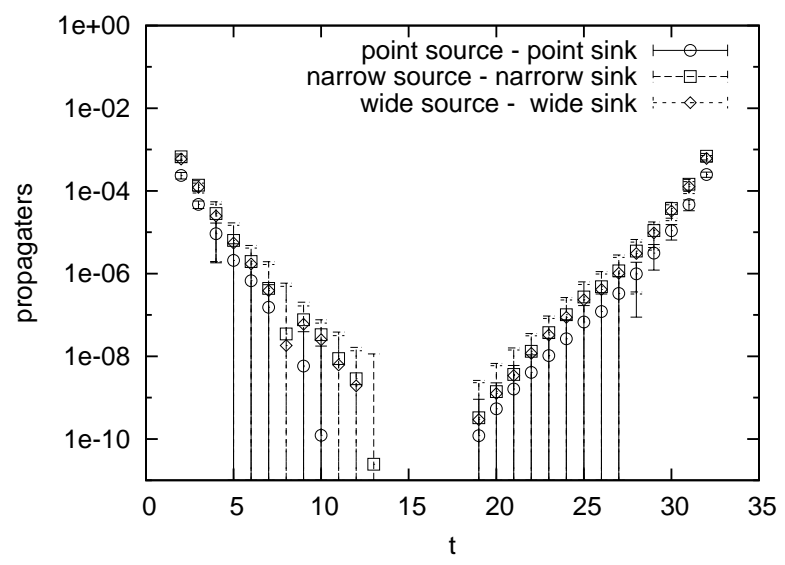

Fig. 9. The $\kappa$ propagaters calculated with same type operaters of source and sink.

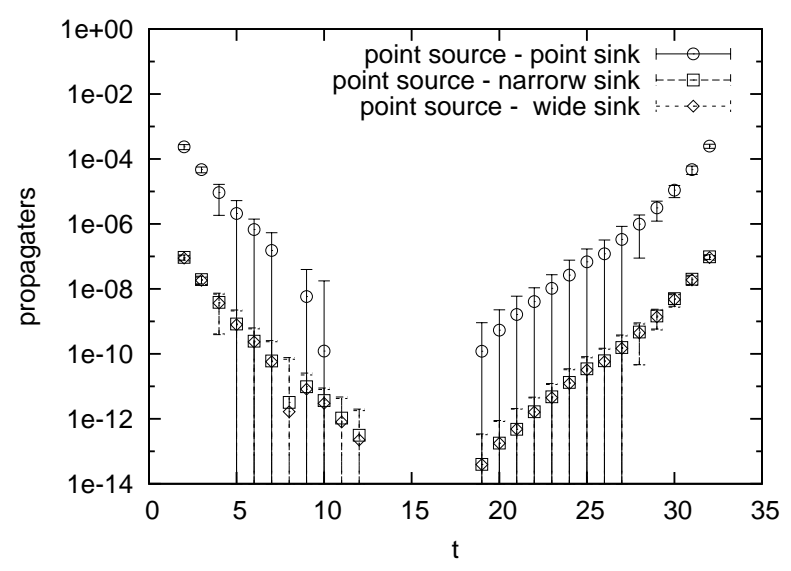

Fig. 10. The $\kappa$ propagaters calculated with different type operaters of source and sink.

\section{Summary}

The $\sigma$ and other low-lying scalar mesons are still a source of debates. All full QCD lattice simulation suggests the existence of a low-lying sigma as a pole in QCD; the physical content is obscure: the disconnected diagram gives the dominate contribution. A quenched lattice calculation suggests that the $\kappa$ meson can not a normal $q \bar{q}$ state. We will present the new results of the $\sigma$ and the $\kappa$ meson in the near future.

The calculation was done on SX-5, SX-8, SX-9 at RCNP, Osaka university and on SR-8000 at KEK. 
$19^{\text {th }}$ International IUPAP Conference on Few-Body Problems in Physics

\section{References}

1. K. Igi and K. i. Hikasa, Phys. Rev. D 59 (1999) 034005.

2. I. Caprini, G. Colangelo and H. Leutwyler, Phys. Rev. Lett. 96 (2006) 132001.

3. Particle Data Group Collaboration, C. Amsler et al., Phys. Lett. B667 , 1 (2008).

4. For example, see Possible existence of the sigmameson and its implications to hadron physics, KEK Proceedings 2000-4, Soryushiron Kenkyu (kyoto)102, E1 (2001).

5. E791 Collaboration, E. M. Aitala et al., Phys. Rev. Lett. 86, 770 (2001).

6. M. Ishida, S. Ishida, T. Kodama, S. Matsumoto, Phys. Lett. B518, 47 (2001).

7. D. B. Bugg, Phys. Lett. B572, 1 (2003).

8. E791 Collaboration, M. Aitala et al., Phys. Rev. Lett. 89, 121801 (2002).

9. BES Collaboration, M. Ablikim et al., Phys. Lett. B633, 681 (2006).

10. F. E. Close and N. A. Törnqvist, J. Phys. G: Nucl. Part. Phys. 28, R249 (2002).

11. R. L. Jaffe, Phys. Rev. D 15267 (1977).

12. M. Alfold and R. L. Jaffe, Nucl. Phys. B578, 367 (2000).

13. Y. Nambu and G. Jona-Lasinio, Phys. Rev. 122, 345 (1961); 124, 246 (1961).

14. T. Hatsuda and T. Kunihiro, Prog. Theor. Phys. 74, 765 (1985); see also T. Hatsuda and T. Kunihiro, Phys. Rep. 247, 221 (1994); T. Kunihiro, Prog. Theor. Phys. Suppl. 120, 75 (1995).

15. W. Lee and D. Weingarten, Phys. Rev. D61, 014015 (1999).

16. C. McNeile and C. Michael, Phys. Rev. D63, 114503 (2001).

17. S. Narison, hep-ph/0009108; Nucl. Phys. B96 (Proc. Suppl.), 244 (2001); 121, 131 (2003).

18. C. DeTar and J. B. Kogut, Phys. Rev. D36 , 2828 (1987).

19. S. Kim and S. Otha Nucl. Phys. Proc. Suppl, 53, 199 (1997), ibid. 63, 185 (1998).

20. C. Hanhart, J. R. Pelaez and G. Rios, Phys. Lett. 100, 152001 (2008).

21. SCALAR Collaboration, S. Muroya et al., Nucl. Phys. Proc. Suppl. 106, 272 (2002).

22. SCALAR Collaboration, T. Kunihiro et al., Nucl. Phys. Proc. Suppl. 119, 275 (2003).

23. SCALAR Collaboration, H. Wada, Nucl. Phys. Proc. Suppl. 129, 432 (2004).

24. SCALAR Collaboration, T. Kunihiro et al., Phys. Rev. D70, 034504 (2004).

25. CP-PACS Collaboration, S. Aoki et al., Phys. Rev. D60, 114508 (1999).

26. UKQCD Collaboration, A. Hart et al., Phys. Rev. D74, 114504 (2006).

27. N. Mathur et al., Phys. Rev. D70, 074508 (2004).

28. H. Suganuma et al., Prog. Theor. Phys. Suppl. 168, 168 (2007).
29. M. Loan, Z.Luo and Y. Y. Lam, Eur. Phys. J. C57 579 (2008).

30. S. Prelovsek et al., arXix:0910.2749[hep-lat].

31. SCALAR Collaboration, T. Kunihiro., Nucl. Phys. Proc. Suppl. 129, 242 (2004).

32. SCALAR Collaboration, H. Wada et al., Phys. Lett. B 652250 (2007).

33. S. Prelovsek, C. Dawson, T. Izubuchi K. Orginos and A. Soni, Phys. Rev. D70, 094503 (2004).

34. UKQCD Collaboration, C. McNeile and C. Michael, Phys. Rev. D74, 014508 (2006).

35. CP-PACS Collaboration, S. Aoki, et al., Phys. Rev. D67, 034503 (2003).

36. C. Gattringer et al., Phys. Rev. D78, 034501 (2008).

37. CP-PACS Collaboration, S. Aoki et al., Phys. Rev D65, 054505 (2002) [E: D67, 059901 (2003)].

38. Trinlat Collaboration, J. Foley, et al., Compt. Phys. Commun. 172 , 145 (2005). 